\title{
Small molecular compounds efficiently convert human fibroblasts directly into neurons
}

\author{
JIJUAN YANG ${ }^{1,2^{*}}$, HUIMEI CAO ${ }^{1 *}$, SHENGNAN GUO $^{1}$, HONG ZHU $^{2}$, HONG TAO $^{1}$,

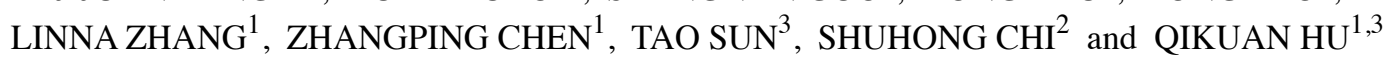 \\ ${ }^{1}$ Department of Physiology, Ningxia Medical University; ${ }^{2}$ Department of Rheumatology, \\ The General Hospital of Ningxia Medical University; ${ }^{3}$ Ningxia Key Laboratory of Cerebrocranial Disease, \\ Basic Medical School of Ningxia Medical University, Yinchuan, Ningxia 750004, P.R. China
}

Received March 5, 2020; Accepted August 14, 2020

DOI: $10.3892 / \mathrm{mmr} .2020 .11559$

\begin{abstract}
No effective treatment is currently available for neurodegenerative diseases, and existing pharmacotherapy is inconsistent with severe side effects. Cell replacement therapy is promising for neurodegenerative disease treatment, and the induction of neurons is an unmet need for such therapy. The present study investigated the potential of a combined medium composed of conditioned medium and eight small molecular compounds in reprogramming human foreskin fibroblasts (HFFs) into neurons. HFFs were cultured from foreskin and then induced by small molecules to generate neurons. The results demonstrated that the conditioned medium containing forskolin, RepSox, SP600125, CHIR99021, Go6983, Y-27632, IXS9 and I-BET151 effectively induced human fibroblasts to change into neurons in vitro. Following a 30-day induction, the cells exhibited neuronal properties as determined by morphological and phenotypical alterations. The induced cells exhibited expression of neuronal markers, including class III $\beta$-tubulin, microtubule-associated protein 2 , vesicular glutamate transporter 1 and $\gamma$-aminobutyric acid, accompanied by increased expression of neuronal transcription factors, including neuronal differentiation 1 and achaete-scute family
\end{abstract}

Correspondence to: Dr Qikuan Hu, Department of Physiology, Ningxia Medical University, 1160 Shengli Street, Yinchuan, Ningxia 750004, P.R. China

E-mail: nxykdxhqk@126.com

Dr Shuhong Chi, Department of Rheumatology, The General Hospital of Ningxia Medical University, 804 South Shengli Street, Yinchuan, Ningxia 750004, P.R. China

E-mail: chi794613@163.com

${ }^{*}$ Contributed equally

Abbreviations: ESCs, embryonic stem cells; HFFs, human foreskin fibroblasts; PBST, PBS with $0.1 \%$ Tween-20; TTX, tetrodotoxin

Key words: small molecular compounds, fibroblasts, neurons, neuronal markers, electrophysiological properties
bHLH transcription factor 1, and decreased expression levels of fibroblast-specific genes. Furthermore, these cells also exhibited electrophysiological properties of neurons. Notably, the course of cell morphological alterations demonstrated the differentiation of fibroblasts into neurons. The present study provided a novel combination of existing small molecular compounds that efficiently reprogramed human fibroblasts into neurons.

\section{Introduction}

Neurodegenerative diseases, such as epilepsy, Alzheimer's disease and Huntington's, are a major global burden to human health. Currently, there are no effective treatments available for the majority of neurodegenerative diseases $(1,2)$, and the existing pharmacotherapy is inconsistent with severe adverse effects. Cell replacement therapy with neurons may be an alternative therapy for these diseases (1). Therefore, in vitro generation and expansion of neurons are critical for the cell therapy approach, although neurons can be generated by direct conversion for disease modeling $(2,3)$. Current efforts are focused on using exogenous cells derived from embryonic stem cells (ESCs) or induced pluripotent stem cells to generate neurons $(4,5)$. However, cell transplantation approaches using stem cell-derived neurons face significant hurdles, such as immunorejection, tumorigenesis, unstable genetic manipulation and differentiation uncertainty $(6,7)$. Therefore, there is a need to find a novel approach for neuronal generation. Small molecular compounds have been used to directly reprogram human fibroblasts into neurons due to their advantages, including being non-immunogenic, lower costs and easy optimization $(8,9)$. Compared with transcription factor-based reprogramming, small molecular compound-mediated trans-differentiation has the potential to evolve into a rapid and direct approach for the generation of customized cell types, due to the availability of somatic cells (mainly skin-derived fibroblasts) and the flexibility to generate autologous target cells (9). Several studies have reported that a combination of small chemical compounds efficiently and directly converts human fibroblasts into functional neurons (10-12). However, these reprogramming strategies still have some limitations, such as low efficiency and time-consuming induction (11). 
Therefore, it is essential to identify more efficient small molecule-induced reprogramming strategies for cell therapies of neurological disorders.

The present study reports a defined combination of eight small molecular compounds capable of directly reprogramming human foreskin fibroblasts (HFFs) into functional neurons following sequential treatment in vitro. Additionally, the present results revealed the course of morphological cell changes during the inductive periods.

\section{Materials and methods}

Cell culture. HFFs were cultured from the foreskin of a 20-year-old man at The General Hospital of Ningxia Medical University (Yinchuan, China) in October 2016. The present study was approved by the Ethics Review Board of Ningxia Medical University (approval no. 2015-049). Written informed consent was obtained from the patient, and written and signed informed consent for publication of the present study was also obtained. Cells were cultured in DMEM with high glucose (4.5 g/l; HyClone; Cytiva) containing $100 \mathrm{U}$ penicillin and $0.1 \mathrm{mg} / \mathrm{ml}$ streptomycin with $10 \%$ FBS (Clark Bioscience) in a humidified atmosphere containing $5 \% \mathrm{CO}_{2}$ at $37^{\circ} \mathrm{C}$. HFFs labeled with green fluorescent protein (GFP) by lentiviral infection (HFF-GFP). Lentiviral vector and Polybrene (final concentration $5 \mu \mathrm{g} / \mathrm{ml}$ ) were purchased from Shanghai GeneChem Co., Ltd., and were used following the manufacturers' protocols.

Cell induction. Plates were coated with Matrigel at room temperature overnight. Then, HFFs were seeded into Matrigel-coated 24 -well plates $\left(2 \times 10^{4}\right.$ cells/well $)$ and cultured overnight. The fibroblast medium was replaced with neuronal induction medium [DMEM/F12:Neurobasal (1:1) with $0.5 \% \mathrm{~N} 2$ supplement, $1 \%$ B27 and $100 \mathrm{mM}$ cAMP], supplemented with $10 \mu \mathrm{M}$ Forskolin (F), $1 \mu \mathrm{M}$ RepSox (R), $10 \mu \mathrm{M}$ SP600125 (S), $3 \mu \mathrm{M}$ CHIR99021 (C), $5 \mu \mathrm{M}$ GO6983 (G), $5 \mu \mathrm{M}$ Y-27632 (Y), $20 \mu \mathrm{M}$ ISX-9 (I) and $2 \mu \mathrm{M}$ I-BET151 (IB), all purchased from PeproTech, Inc. Subsequently, culture media (neuronal induction media plus the small molecular compounds) were refreshed every 3-4 days during the induction period. Following small molecular compound treatment for 7 days, induction media were changed to neuronal maturation media [the neuronal induction medium plus $20 \mathrm{ng} / \mathrm{ml}$ basic fibroblast growth factor (bFGF), 1\% GlutaMAX, $20 \mathrm{ng} / \mathrm{ml}$ brain-derived neurotrophic factor (BDNF), $20 \mathrm{ng} / \mathrm{ml}$ glial cell line-derived neurotrophic factor (GDNF) and $20 \mathrm{ng} / \mathrm{ml}$ neurotrophin-3 (NT3), all purchased from PeproTech, Inc.], supplemented with $\mathrm{C}+\mathrm{F}+\mathrm{Y}+\mathrm{I}$. Neuronal maturation media were changed every other day. Small molecular compounds were dissolved and diluted in dimethyl sulfoxide (Sigma-Aldrich; Merck $\mathrm{KGaA}$ ) according to the manufacturer's protocols and then processed to use at the following final concentrations: $10 \mu \mathrm{M} \mathrm{F}, 1 \mu \mathrm{M} \mathrm{R}, 10 \mu \mathrm{M} \mathrm{S}, 3 \mu \mathrm{M} \mathrm{C}, 5 \mu \mathrm{M} \mathrm{G}, 5 \mu \mathrm{M} \mathrm{Y}$, $20 \mu \mathrm{M} \mathrm{I}, 2 \mu \mathrm{M}$ IB, $0.5 \mathrm{mM}$ valproic acid (VPA) and $1 \mu \mathrm{M}$ Dorsomorphin (D). The concentrations of small molecules were selected on the basis of previous literatures (8). All small molecular compounds were purchased from Stemcell Technologies, Inc.
Immunofluorescent staining. Cells were grown on coverslips and fixed with $4 \%$ formaldehyde for $10 \mathrm{~min}$ at room temperature and subsequently washed with PBS containing $0.1 \%$ Tween-20 (PBST). After blocking at room temperature with 5\% normal goat serum (Beijing Solarbio Science \& Technology Co., Ltd.) in PBST for 30 min, cells were incubated with the primary antibody overnight at $4^{\circ} \mathrm{C}$, then washed with PBST, and finally incubated with a goat anti-mouse $\mathrm{IgG}$ Alexa Fluor 555-conjugated secondary antibody (1:1,000; cat. no. A-21422; Thermo Fisher Scientific, Inc.) for $1 \mathrm{~h}$ at room temperature. Cells were observed and imaged using laser scanning confocal microscopy (Fluoview FV10; Olympus Corporation) and an inverted fluorescence microscope (TS100; Nikon Corporation). The primary antibodies used in the present study were as follows: mouse anti-class III $\beta$-tubulin (Tuj1; 1:250; cat. no. 480011; Thermo Fisher Scientific, Inc.), rabbit anti-microtubule-associated protein 2 (MAP2; 1:200; cat. no. 17490-1-AP; ProteinTech Group, Inc.), rabbit anti-vesicular glutamate transporter 1 (vGLUT1; 1:1,000; cat. no. 48-2400; Thermo Fisher Scientific, Inc.), rabbit anti- $\gamma$-aminobutyric acid (GABA; 1:500; cat. no. PA5-32241; Thermo Fisher Scientific, Inc.), rabbit anti-neuronal nuclei (NeuN; 1:200; cat. no. 26975-1-AP; ProteinTech Group, Inc.), rabbit anti-OCT4 (1:500; cat. no. MA1-104; Thermo Fisher Scientific, Inc.) and rabbit anti-glial fibrillary acidic protein (GFAP; 1:200; cat. no. 16825-1-AP; ProteinTech Group, Inc.). Semi-quantification was performed using ImageJ software (version 1.44; National Institutes of Health).

Reverse transcription-quantitative PCR (RT-qPCR). Total RNA was extracted from cells using the RNAsimple Total RNA kit (Tiangen Biotech Co., Ltd.) and reverse transcribed to cDNA using the TIANScript RT kit (Tiangen Biotech Co., Ltd.) according to the manufacturers' protocols. Thermocycling conditions for reverse transcription were as follows: $42^{\circ} \mathrm{C}$ for $60 \mathrm{~min}$ and $85^{\circ} \mathrm{C}$ for $5 \mathrm{~min}$. The mRNA expression levels were quantified by SuperReal PreMix Plus (SYBR-Green) kit (Tiangen Biotech Co., Ltd.) using a Bio-Rad CFX Manager 3.0 (Bio-Rad Laboratories, Inc.). Thermocycling conditions for qPCR were as follows: $95^{\circ} \mathrm{C}$ for $15 \mathrm{~min}$, followed by 40 cycles of $95^{\circ} \mathrm{C}$ for $10 \mathrm{sec}, 60^{\circ} \mathrm{C}$ for $30 \mathrm{sec}$. Results were confirmed in at least three separate analyses. The sequences of gene primers are shown in Table I. The relative mRNA levels were calculated using the $2^{-\Delta \Delta \mathrm{Cq}}$ method (13) and normalized to GAPDH.

Electrophysiological analysis. For whole-cell patch-clamp recordings, the artificial cerebrospinal fluid extracellular solution contained $140 \mathrm{mM} \mathrm{NaCl}, 5 \mathrm{mM} \mathrm{KCl}, 1 \mathrm{mM} \mathrm{MgCl}$, $1.8 \mathrm{mM} \mathrm{CaCl}, 1.25 \mathrm{mM} \mathrm{NaH} \mathrm{PO}_{4}, 23 \mathrm{mM}$ glucose and $10 \mathrm{mM}$ HEPES (pH adjusted to 7.4 with $\mathrm{NaOH}$ ). Patch pipettes (3-5 M $\Omega$ ) were filled with an internal solution containing $93 \mathrm{mM}$ K-gluconate, $16 \mathrm{mM} \mathrm{KCl}, 2 \mathrm{mM} \mathrm{MgCl}{ }_{2}, 10 \mathrm{mM}$ HEPES, $10 \mathrm{mM}$ Na-phosphocreatine, $0.3 \mathrm{mM}$ GTP-Na and $4 \mathrm{mM}$ ATP-Mg. Sodium currents were recorded using the voltage clamp method. Step voltages with an increment of $10 \mathrm{mV}$ were injected to elicit an inward sodium current. To block the sodium current, $1 \mu \mathrm{M}$ tetrodotoxin (TTX) was added into the chamber, and step voltages were injected again to observe the TTX-sensitive currents $10 \mathrm{~min}$ after applying TTX. Patch pipettes were pulled with a P97 micropipette 
Table I. Primer sets for reverse transcription-quantitative PCR.

\begin{tabular}{|c|c|}
\hline Gene name & Sequence $\left(5^{\prime} \rightarrow 3^{\prime}\right)$ \\
\hline CTGF & $\begin{array}{l}\text { F: GCGAAGCTGACCTGGAAGAGAAC } \\
\text { R: GCTCGGTATGTCTTCATGCTGGTG }\end{array}$ \\
\hline COL1A1 & $\begin{array}{l}\text { F: AGAACGCCAAGGACAAGAAGCAC } \\
\text { R: CCATCAGACGCAGGAAGGTAAGC }\end{array}$ \\
\hline DKK3 & $\begin{array}{l}\text { F: AGCTATCACAATGAGACCAACACAGAC } \\
\text { R: GATGATGCACTCGTGGCTCCTTC }\end{array}$ \\
\hline NeuroD1 & $\begin{array}{l}\text { F: CCTCGAAGCCATGAACGCAGAG } \\
\text { R: TCGTCATCCTCCTCTTCCTCTTCTTC }\end{array}$ \\
\hline ASCL1 & $\begin{array}{l}\text { F: ACAAGAAGATGAGTAAGGTGGAGACAC } \\
\text { R: ATGGAGTTCAAGTCGTTGGAGTAGTTG }\end{array}$ \\
\hline DCX & $\begin{array}{l}\text { F: GGATTGTGTACGCTGTGTCCTCTG } \\
\text { R: TTCATCCATGCTTCCGATCTTCCTG }\end{array}$ \\
\hline MAP2 & $\begin{array}{l}\text { F: TTGGTGCCGAGTGAGAAGAA } \\
\text { R: AGGTCTGGCAGTGGTTGGTT }\end{array}$ \\
\hline GAPDH & $\begin{array}{l}\text { F: CAGGAGGCATTGCTGATGAT } \\
\text { R: GAAGGCTGGGGCTCATTT }\end{array}$ \\
\hline
\end{tabular}

ASCL1, achaete-scute family bHLH transcription factor 1; COL1A1, collagen type $1 \alpha-1$ chain; CTGF, connective tissue growth factor; DCX, doublecortin; DKK3, dickkopf WNT signaling pathway inhibitor 3; MAP2, microtubule-associated protein 2; NeuroD1, neuronal differentiation $1 ; \mathrm{F}$, forward; $\mathrm{R}$, reverse.

puller to achieve a 3-6 $\mathrm{M} \Omega$ tip resistance. Patch-clamp recordings were taken using an EPC-10 amplifier (HEKA Elektronik $\mathrm{GmbH}$; Harvard Bioscience, Inc.) with PatchMaster 2.90 (HEKA Elektronik GmbH; Harvard Bioscience, Inc.).

Western blot analysis. Cells were washed twice in PBS and proteins were extracted with RIPA lysis buffer (Beyotime Institute of Biotechnology) supplemented with protease inhibitor cocktail (cat. no. P1008; Beyotime Institute of Biotechnology) and boiled at $100^{\circ} \mathrm{C}$ for $5 \mathrm{~min}$. Protein concentrations were measured by a bicinchoninic acid assay (cat. no. P0012S; Beyotime Institute of Biotechnology). Subsequently, $80 \mu \mathrm{g}$ whole-cell extract proteins were separated on $10 \%$ SDS-polyacrylamide gels, and transferred to a polyvinylidine difluoride membrane (EMD Millipore). Non-specific binding was blocked by incubation in 5\% skimmed milk in PBST for $2 \mathrm{~h}$ at room temperature. Membranes were incubated at $4^{\circ} \mathrm{C}$ overnight with rabbit anti- $\beta \amalg$-tubulin $(1: 1,000$; cat. no. ab18207; Abcam), rabbit anti-Synapsin 1 (1:1,000; cat. no. PA1-4673; Thermo Fisher Scientific, Inc.) and rabbit anti-NeuN (1:1,000; cat. no. 26975-1-AP; ProteinTech Group, Inc.). Afterwards, the membranes were washed in PBST and were incubated with appropriate fluorescently labeled secondary antibodies for $1 \mathrm{~h}$ at room temperature. The secondary antibodies used were: goat anti-rabbit (IRDye ${ }^{\circledR}$ 680RD; 1:5,000; cat. no. 925-68071; LI-COR Biosciences) and goat anti-mouse (IRDye ${ }^{\circledR} 680 \mathrm{RD}$; 1:6,000; cat. no. 925-68070; LI-COR Biosciences). The membranes were scanned using the Odyssey infrared imaging system (LI-COR Biosciences), and protein band intensity was semi-quantified by computerized densitometry using ImageJ software 1.46r (National Institutes of Health) and expressed as arbitrary units after normalization to GAPDH (1:5,000; cat. no. PA1-988; Thermo Fisher Scientific, Inc.).

Transmission electron microscopy. Cells were collected and washed briefly with a $0.1 \mathrm{M}$ sodium cacodylate buffer prior to fixation with $2.5 \%$ glutaraldehyde in $0.1 \mathrm{M}$ sodium cacodylate overnight at $4^{\circ} \mathrm{C}$. After being rinsed for $10 \mathrm{~min}$ in the same buffer, the cells were fixed at $4^{\circ} \mathrm{C}$ for $1 \mathrm{~h}$ with $1 \% \mathrm{OsO}_{4}$ in $0.1 \mathrm{M}$ sodium cacodylate. Following dehydration with a standard ethanol series and infiltration in epoxy resin at room temperature overnight, cells were transferred to beam capsules for polymerization in the oven $\left(35^{\circ} \mathrm{C}\right.$ for $6 \mathrm{~h}, 42^{\circ} \mathrm{C}$ for $18 \mathrm{~h}$ and $60^{\circ} \mathrm{C}$ for $48 \mathrm{~h}$ ). The capsules were separated from the polymerized resin with a razor blade, and embedded cells in hardened blocks were viewed with an optical microscope so that the appropriate area was chosen for ultrathin sectioning $(75 \mathrm{~nm}$ thick). Subsequently, ultrathin sections were obtained using an ultramicrotome with a diamond knife. Heavy metal staining was performed at room temperature for $30 \mathrm{~min}$ with $4 \%$ uranyl acetate and lead citrate, and the samples were examined under an electron microscope (H-7800; Hitachi, Ltd.).

Mitochondrial staining. Cells were grown in confocal Petri dishes filled with the appropriate culture medium. When cells reached the desired confluence $(\sim 40 \%)$, an equal volume of the dye-working solution (AAT Bioquest, Inc.) was added. The cells were incubated in a $5 \% \mathrm{CO}_{2}$ incubator at $37^{\circ} \mathrm{C}$ for $1 \mathrm{~h}$. The cell nucleus was stained with Hoechst 33342 (Sigma-Aldrich; Merck $\mathrm{KGaA}$ ) at $37^{\circ} \mathrm{C}$ for $30 \mathrm{~min}$. The cells were observed using a laser scanning confocal microscope (Fluoview FV10; Olympus Corporation).

Statistical analysis. Data are presented as the mean \pm standard deviation, and experiments were performed at least three times. Statistical analysis was performed using SPSS 17.0 software (SPSS, Inc.) and data were assessed for normality and variance. Data were compared using an unpaired Student's t-test or one-way analysis of variance with a Bonferroni post hoc test. $\mathrm{P}<0.05$ was considered to indicate a statistically significant difference.

\section{Results}

Screening for optimal small molecular compounds. The present study was designed to refine small molecular compounds able to reprogram HFFs into functional neurons. The initial HFFs derived from the foreskin of a 20 -year-old man showed no contamination of neuronal cells or neural stem cells (Fig. 1A). A total of 11 small molecular compounds were initially included as the starting candidate pool based on three major criteria of selection that a compound has: i) the capacity to disrupt fibroblast-specific programs, such as IB (9); ii) the ability to activate neural reprogramming related signaling pathways, to enhance neuronal gene expression, to promote neuronal conversion of human fibroblasts and reprogramming efficiency, such as valproic acid (VPA), F, C, G, S, I, purmorphamine $(\mathrm{P})$ and $\mathrm{D}(8,9,14-16)$; and iii) the potential to promote 

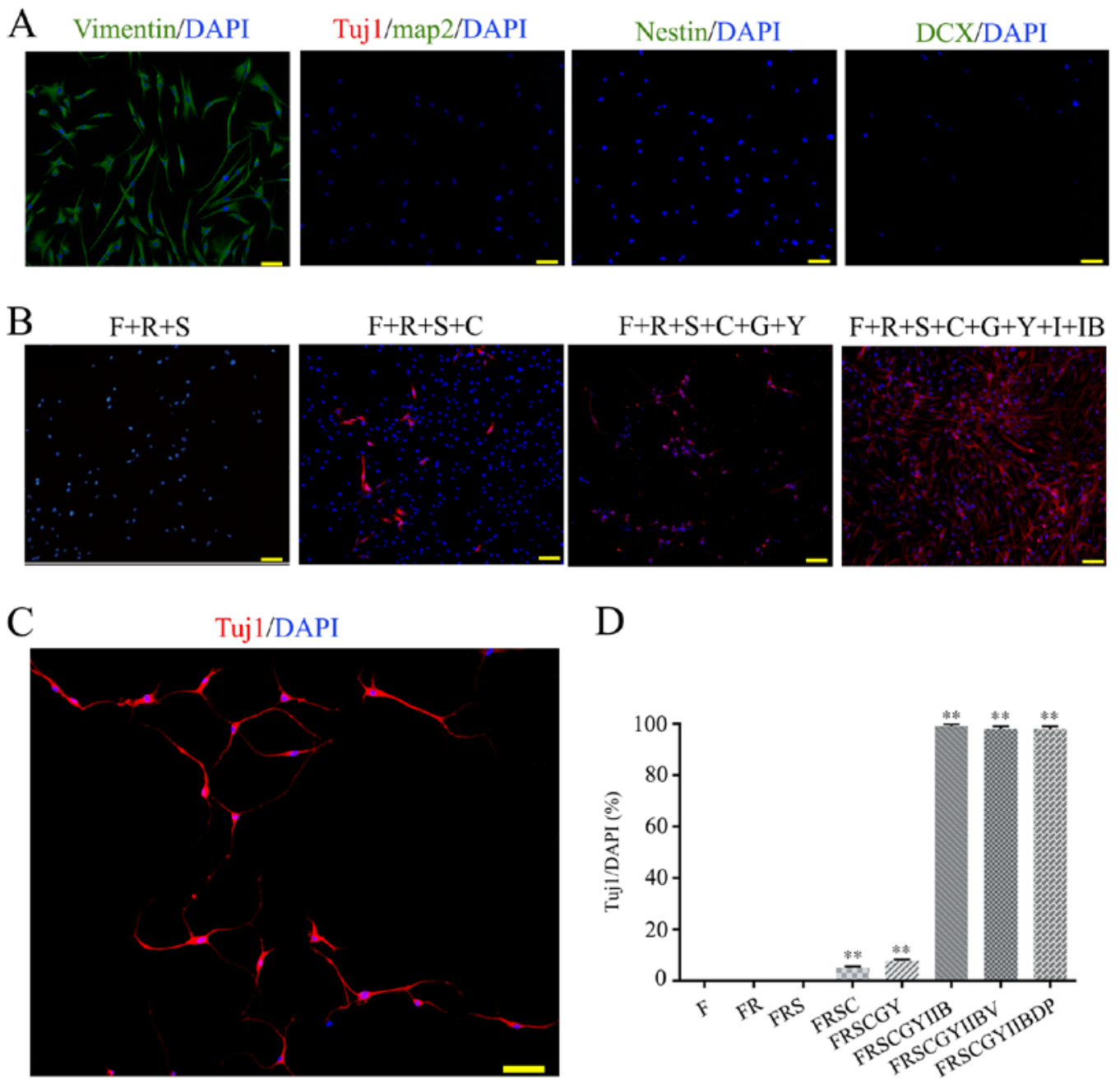

$\mathrm{D}$

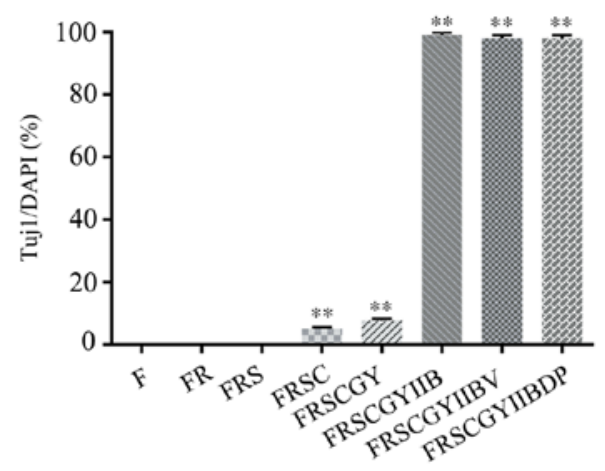

Figure 1. Characterization of HFFs and optimal small molecule induction protocol. (A) Immunofluorescence staining of the HFFs. Fibroblast marker, vimentin; neuronal markers, MAP2, Tuj1, nestin and DCX. (B) Optimal small molecule combination. Immunofluorescence staining of HFFs reprogrammed with F, R, S, C, G, Y, I and IB on day 4. (C) Immunofluorescence staining of Tuj1 (red) on day 7. (D) Percentage of Tuj1 ${ }^{+} /$DAPI after treatment of HFFs with different small molecular compounds. Cells from at least five random fields of view in each of the three wells from three independent experiments were counted. Scale bar, $50 \mu \mathrm{m}$. Cell nuclei were stained with DAPI. Data are presented as the mean \pm SD. ${ }^{* *} \mathrm{P}<0.01$ vs. F only group. F, forskolin; R, RepSox; S, SP600125; C, CHIR99021; G, Go6983; Y, Y-27632; I, IXS9; IB, I-BET151; Tuj1, class III $\beta$-tubulin; DCX, doublecortex; MAP2, microtubule-associated protein 2; HFF, human foreskin fibroblast.

neuronal survival, such as $\mathrm{R}$ and $\mathrm{Y}(15,17)$. The HFFs were treated with a neuronal induction medium containing $\mathrm{F}, \mathrm{F}+\mathrm{R}$ and $\mathrm{F}+\mathrm{R}+\mathrm{S}$, and no neuron-specific marker Tuj1-positive cells were detected on day 4 (Fig. 1B). The HFFs were treated with $\mathrm{F}+\mathrm{R}+\mathrm{S}+\mathrm{C}$, an agent suggested to improve neuronal conversion of HFFs $(8,18)$, and $5 \%$ of cells expressed Tuj1 (Fig. 1B). In order to improve the induction efficiency, small molecular compounds $\mathrm{G}+\mathrm{Y}$ were supplemented based on the original $\mathrm{F}+\mathrm{R}+\mathrm{S}+\mathrm{C}$. The number of Tuj1-positive cells was increased to $8 \%$ in this medium, but the cells did not present a typical neuronal morphology (Fig. 1B), which suggested that they were inefficiently reprogrammed (Fig. 1B). Notably, almost all cells expressed the neuronal maker Tuj1 following treatment with eight small molecular compounds $(\mathrm{F}+\mathrm{R}+\mathrm{S}+\mathrm{C}+\mathrm{G}+\mathrm{Y}+\mathrm{I}+\mathrm{IB})$ on day 4 (Fig. 1B). Under these conditions, the induced cells exhibited a bipolar neuron-like cell morphology, particularly on day 7 (Fig. 1C). Notably, there was no significant difference in the number of both Tuj1-positive and DAPI-positive cells between those cultured in a medium with or without $\mathrm{V}, \mathrm{P}$ and D. Therefore, compounds V, D and P were removed from the combination of small molecular compounds (Fig. 1D). In order to promote neuronal maturation following fibroblast-neuron conversion, compounds $\mathrm{R}+\mathrm{S}+\mathrm{G}+\mathrm{IB}$ were also removed from the medium and replaced with neurotrophic factors (BDNF, GDNF and NT3) on day 7 of culture. Finally, a cocktail containing eight small molecular compounds ( $F, R$, S, C, G, Y, I and IB) was used to reprogram human fibroblasts into neurons, and the compounds were added into the culture medium in a stepwise manner.

Morphological alterations of cells during induction. When recording the phenotypical changes of cells between days 1 and 30 of induction, gradual morphological changes of the HFFs from fibroblasts to typical neurons were observed at $12 \mathrm{~h}$ after the addition of small molecular compounds (Fig. 2A). The cells then underwent robust morphological changes with a decreased cell volume and pervasive neurite extension during the first $24 \mathrm{~h}$ of induction, as determined by a time course live cell imaging (Fig. 2C). Due to the long-term reprogramming progression, the induced cells were designated 
A

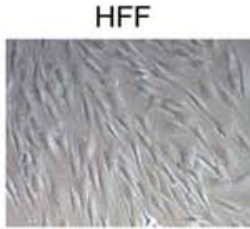

Day 7
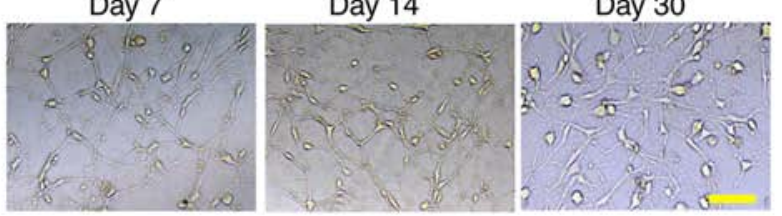

C
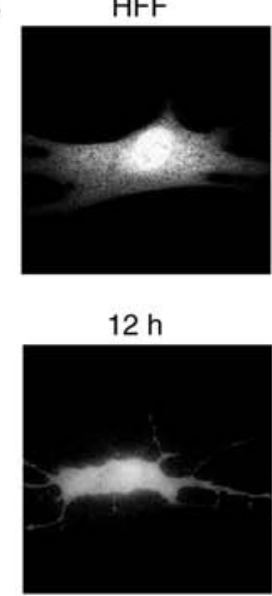

Day 14

$12 \mathrm{~h}$

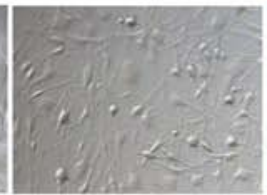

Day 14

$3 \mathrm{~h}$

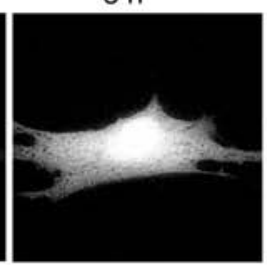

$24 \mathrm{~h}$

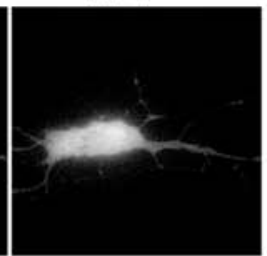

Day 4

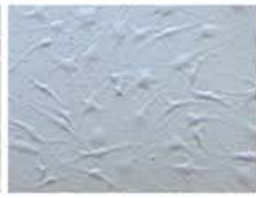

Day 30

$6 \mathrm{~h}$

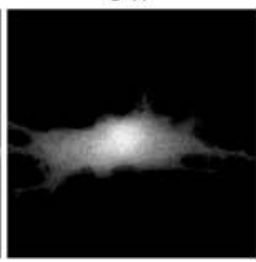

$30 \mathrm{~h}$

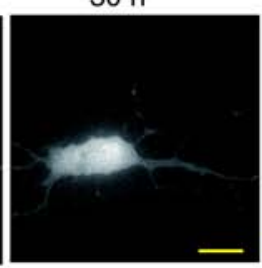

B

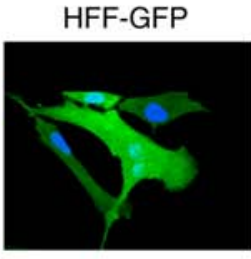

Day 5

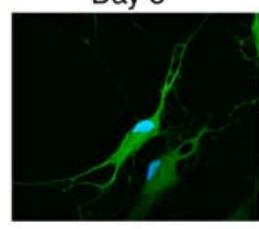

$12 \mathrm{~h}$

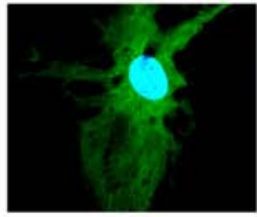

Day 7

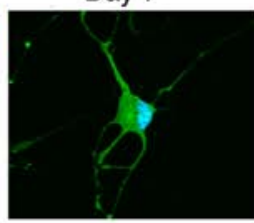

Day 3

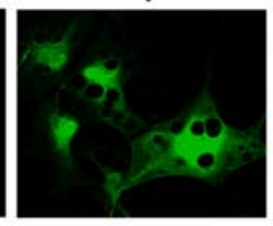

Day 9

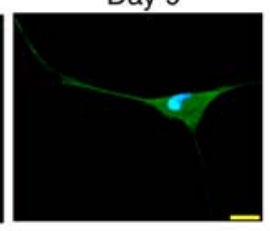

$\mathrm{D}$

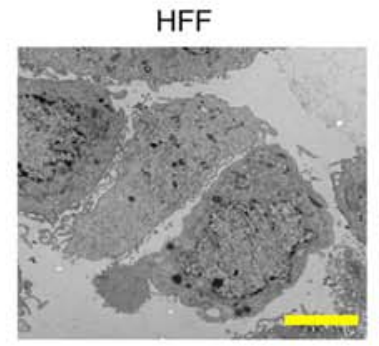

Day 3

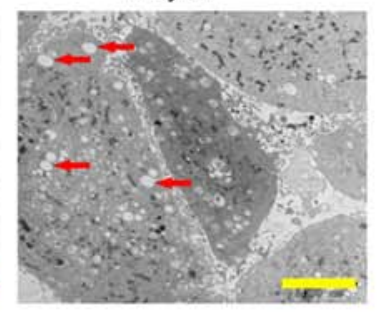

Figure 2. Morphological alterations of cells during induction. (A) Cell morphological changes in bright field microscopy between $12 \mathrm{~h}$ and $30 \mathrm{days}$ after treatment of HFFs with small molecular compounds. Scale bar, $50 \mu \mathrm{m}$. (B) During the reprogramming process, small holes gradually appeared in the cytoplasm of the HFF-GFP cells, and the holes gradually expanded and merged and finally disappeared on day 9 (red arrows). Nucleus stained with Hoechst. Scale bar, $20 \mu \mathrm{m}$. (C) HFFs were treated with small molecule compounds, and single cells were recorded by live cell technique for $30 \mathrm{~h}$ to reveal that fibroblasts rapidly changed to neuron-like morphology. Scale bar, $50 \mu \mathrm{m}$. All images are representative of three independent experiments (n=3). (D) Electron microscopy revealed no holes in HFF-GFP (left panel). Electron microscopy revealed round holes of different sizes in the cytoplasm of cells after 3 days of induction (right panel; black arrows indicate holes). Scale bar, $500 \mu \mathrm{m}$. GFP, green fluorescent protein; HFF, human foreskin fibroblast.

as induced neurons (iNs) in the present study. The iNs exhibited a typical neuronal phenotype on day 7 (Fig. 1C). Notably, HFFs labeled with GFP by lentiviral infection (HFF-GFP) showed small circular blebs in the cytoplasm at $12 \mathrm{~h}$ post induction, which reached the peak on day 3 (Fig. 2B). With the increased number of blebs, small blebs gradually merged into large blebs in the cytoplasm before they disappeared on day 9 (Fig. 2B). This finding was further confirmed by electron microscopy (black arrows; Fig. 2D).

Reprogramming human fibroblasts into neurons using small molecular compounds. After demonstrating that small molecular compounds can induce HFFs to express Tuj1, a cocktail of eight small molecular compounds was demonstrated to induce cells to express Tuj1 and MAP2 with typical neuron-like morphology by day 7 (Figs. 1C and 3A). Most of these iNs were simultaneously expressing Tuj1 and MAP2, accompanied by an extensive neurite outgrowth following 1-2 weeks of induction (Fig. 3A and B). The efficiency of conversion from fibroblasts into neurons induced by this combination was at most, $>92 \%$ and $82.66 \pm 2.52 \%$ after 7-14 and 14-30 days of induction, respectively (data not shown). This suggests an efficient conversion acquired by the combination of small molecular compounds. As the senescence of neurons tended to occur at maturation at $\sim 7$ days, the inductive medium was replaced with the neuronal maturation medium supplemented with $\mathrm{C}+\mathrm{F}+\mathrm{Y}+\mathrm{I}$ and extra neurotropic factors (BDNF, GDNF and NT3) 9 days after the initial conversion. This was able to sustain neuronal cell survival for $>30$ days, as determined by immunofluorescent staining of neuronal markers Tuj1, MAP2, NeuN, GABA and vGLUT1 (Fig. 3B). However, the expression levels of astrocyte marker GFAP and neural stem cell marker OCT4 were undetectable (data not shown).

To further characterize the iN phenotype, the expression levels of four neuron-specific genes and three human fibroblast-specific genes were determined by RT-qPCR in cells cultured for 30 days following small molecular compound treatment. Compared with control HFF (treated with DMSO), a significant increase in the expression levels of several neuron-specific genes, including MAP2, doublecortin, neuronal differentiation 1 (NeuroD1) and achaete-scute family bHLH transcription factor 1 (Ascl1), was observed (Fig. 3C). By contrast, significantly decreased expression levels of human fibroblast-specific genes, including dickkopf WNT signaling pathway inhibitor 3 , connective tissue growth factor and collagen type $1 \alpha-1$ chain, were also identified (Fig. 3C). 
A

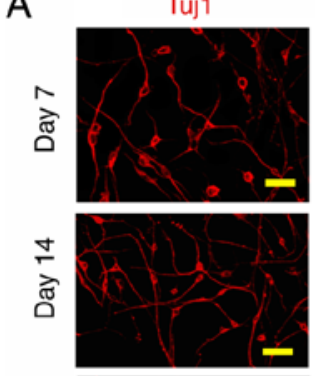

을

ฮิ

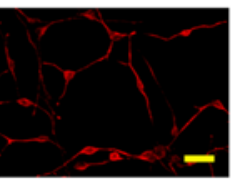

MAP2
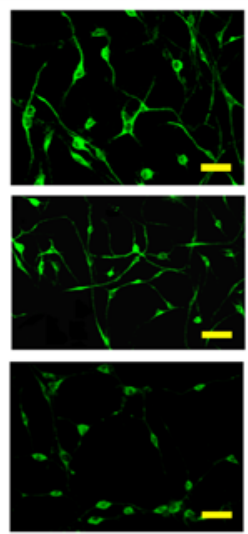

Tuj1/MAP2/DAPI
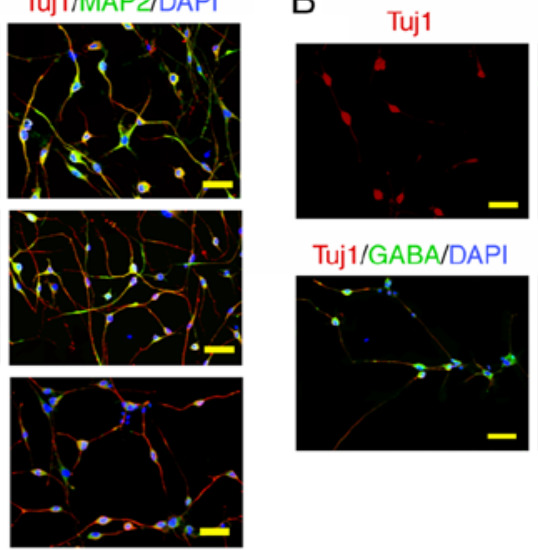

$\mathrm{B}$

Tuj1/GABA/DAPI

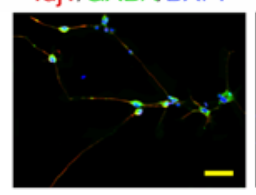

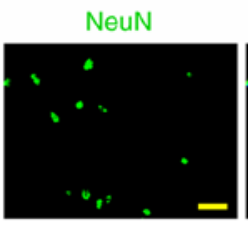

Tuj1/vGLUT1/DAPI

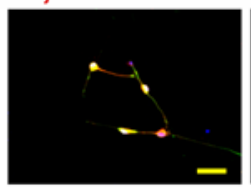

Tuj1/NeuN/DAPI

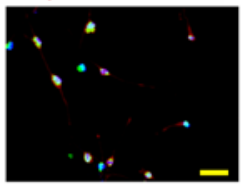

Tuj1/GFAP/DAPI

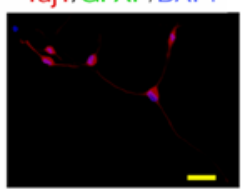

C

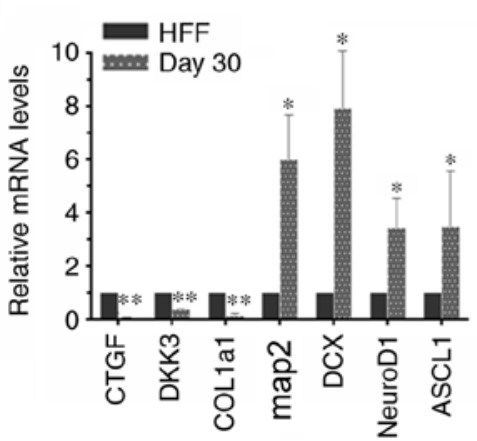

D

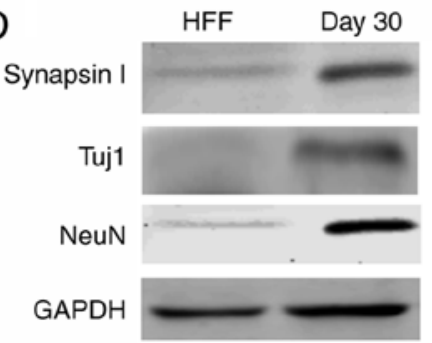

E

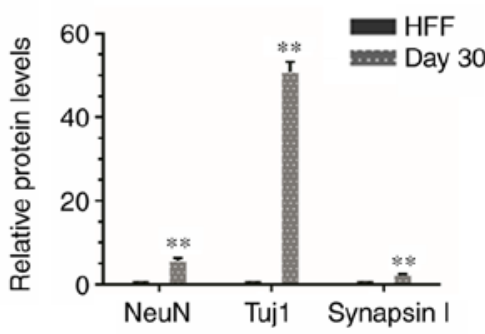

Figure 3. Characteristics of iNs. (A) iNs exhibited bipolar neuronal morphologies and expressed tuj1 (red) and MAP2 (green) at different phases (day 7, 14 and 30). (B) iNs were stained for NeuN, GABA, vGLUT1 and GFAP (all green) on day 30. Scale bar, $50 \mu \mathrm{m}$. (C) Reverse transcription-quantitative PCR analysis revealed that in comparison with HFFs, there was a significant increase in gene expression levels of MAP2, DCX, NeuroD1 and ASCL1 in iNs, whereas human fibroblast-specific genes were downregulated in iNs on day 30. Data are presented as the mean \pm SD. (D) Western blot analysis of the protein expression levels of Tuj1, NeuN and GAPDH in HFFs and iNs. (E) Graphs show the semi-quantification of the western blot analysis. $\mathrm{n}=3$ independent experiments. Data are presented as the mean \pm SD. Unpaired Student's t-tests were used to compare data. ${ }^{*} \mathrm{P}<0.05$, ${ }^{* *} \mathrm{P}<0.01$ vs. HFF. iNs, induced neurons; Tuj1, class III $\beta$-tubulin; MAP2, microtubule-associated protein 2; HFF, human foreskin fibroblast; NeuN, neuronal nuclei; GABA, $\gamma$-aminobutyric acid; vGLUT1, vesicular glutamate transporter 1; GFAP, glial fibrillary acidic protein; DCX, doublecortin; Ascl1, achaete-scute family bHLH transcription factor 1; CTGF, connective tissue growth factor; DKK3, dickkopf WNT signaling pathway inhibitor 3; COL1a1, collagen type $1 \alpha-1$ chain.

Several lines of evidence have demonstrated that fibroblasts are able to directly transdifferentiate into functional neurons using transcription factors $(19,20)$. As expected, 4-fold higher transcription levels of NeuroD1 and Ascl1 genes were gradually increased with the induction course with a peak on day 7 , as compared with untreated HFFs (Fig. 3C). In addition, the alterations in neuronal marker expression were also demonstrated at the protein expression levels by immunoblotting assays in iNs after 30 days of induction (Fig. 3D and E). Overall, these results indicated an activation of neuron-specific genes and inhibition of fibroblast-specific genes in cells exposed to the cocktail of small molecular compounds, suggesting that a combination of small molecular compounds $(\mathrm{F}+\mathrm{R}+\mathrm{S}+\mathrm{C}+\mathrm{G}+\mathrm{Y}+\mathrm{I}+\mathrm{IB})$ was able to efficiently convert HFFs into neurons and to bypass the pluripotent stem stage.

Physiological properties of iNs. To assess whether the iNs from HFFs possess electrophysiological properties, such as the induction of a membrane current, electrophysiological recordings of neurons at different induction stages were performed. None of the cells exposed to the treatment compounds exhibited functional membrane properties after 8 days of induction (Fig. 4A). However, the inward and outward currents could be recorded in cells after 12 days of induction, although the recorded curve was not a typical sodium current and potassium current curve (Fig. 4B). Typical curves of fast inward currents and outward currents were recorded in cells at day 14 post-induction, which might correspond to the opening of voltage-dependent $\mathrm{K}^{+}$and $\mathrm{Na}^{+}$channels (Fig. 4C). Furthermore, the inward current could be completely blocked by the sodium channel-specific inhibitor TTX (Fig. 4D), which indicated that the iNs had functional membrane properties.

\section{Discussion}

Previous studies have demonstrated that fibroblasts can be directly converted into functional neuronal stem cells, neurons, neural progenitors and astrocytes by upregulation of specific transcription factors or microRNAs (21-24). However, inefficient induction and integration of exogenous genes into the genome limit their translation into clinical applications. Small molecular compounds that target signaling pathways, epigenetic modifications or metabolic processes exhibit abilities to regulate cell development, cell fate and function $(9,10,25)$. Therefore, small molecular compounds (chemical reprogramming) that induce cell lineage reprogramming have been widely favored due to their non-immunogenicity, low cost and easy optimization (8). Several studies have reported that 
A

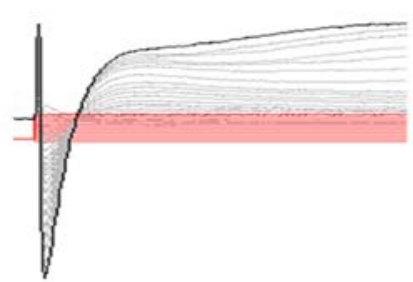

Day 8

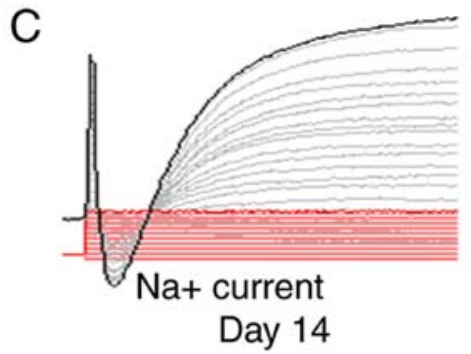

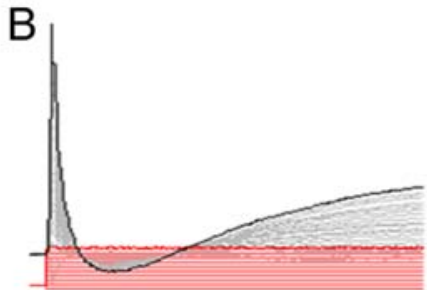

Day 12

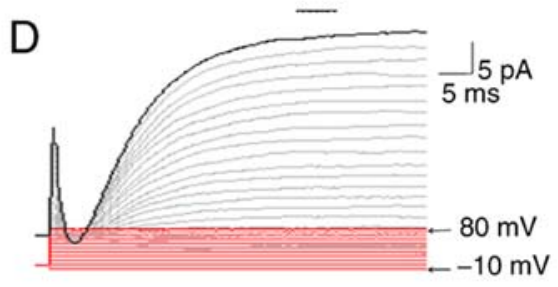

Day 14

Figure 4. Induced neurons have electrophysiological properties of functional neurons. (A) No inward or outward currents were recorded in induced cells on day 8. (B) Inward and outward currents were recorded in the induced cells on day 12, but these were not similar to the electrophysiological characteristics of typical sodium and potassium currents. (C) Representative traces of TTX-sensitive fast inward currents recorded and outward currents in voltage-clamp mode from induced cells on day 14. (D) TTX (1 mM) treatment inhibited voltage-dependent sodium currents. Cells were depolarized from -10 to $80 \mathrm{mV}$ in 10 -mV increments. TTX, tetrodotoxin.

small molecular compounds induce fibroblasts to differentiate into neurons (8-10). However, the induction efficiency and cell morphological changes during induction remain unclear. It is well known that human fibroblasts are bulky, mostly fusiform or star-shaped flat structures, while neurons are relatively small in volume and have long axons (10). The transformation from a relatively large fibroblast into a long axon neuron needs to be observed. The present study first used a cocktail of small molecular compounds that are able to efficiently induce a direct lineage reprogramming from human fibroblasts into neurons. These iNs possessed neuronal properties in terms of gene expression pattern and electrophysiological functional capabilities. Furthermore, the course of morphological changes of cells was recorded during the induction by live cell imaging under a fluorescent microscope.

The cAMP signaling pathway may reduce the barriers of reprogramming by altering downstream epithelial gene expression, decreasing mesenchymal gene expression and increasing cell proliferation (26). It has been reported that activation of the cAMP signaling pathway by forskolin sufficiently generates OCT4-positive colonies in the absence of the OCT4 transgene and replaces OCT4 to induce pluripotency $(26,27)$. $\mathrm{R}$ can be used to replace Sox 2 and c-Myc via inhibition of the TGF- $\beta$ signaling pathway (28). CHIR99021, an agonist of WNT- $\beta$-catenin signaling, promotes ESC self-renewal by potentially upregulating transcription of the protein signal transducer and activator of transcription-3 (29), and promotes the conversion of fibroblasts into neurons $(8,9)$. A combination of F, C, R, S and G has been demonstrated to successfully differentiate fibroblasts into neurons (8). In addition, IB disrupts the fibroblast-specific program, while the neurogenesis inducer I is indispensable to activate neuron-specific genes (9). $\mathrm{Y}$ is able to increase primary keratinocyte proliferation and immortalization without detectable cell senescence (30). Based on previous knowledge regarding the transformation of human fibroblasts into neurons, a cocktail containing eight small molecular compounds $(\mathrm{F}+\mathrm{R}+\mathrm{S}+\mathrm{C}+\mathrm{G}+\mathrm{Y}+\mathrm{I}+\mathrm{IB})$ was used for the induction of HFFs into functional neurons. VPA has been demonstrated to enhance the efficiency of the OCT4, Sox2, Kruppel-like factor 4 (Klf4) and c-Myc four-factor reprogramming mouse fibroblasts $(31,32)$. Despite this, VPA did not appear to serve a notable role in the present study.

In addition, the morphological changes of cells during direct conversion induced by small molecular compounds were recorded in the present study. The morphology of the cells changed markedly; in particular, the cell bodies gradually shrank and the axons gradually formed with time. Additionally, there were differences in the number and structural distribution of mitochondria, in comparison with fibroblasts. The major functions of mitochondria are aerobic energy production, reactive oxygen species production, calcium homeostasis, cellular signaling pathways, and synthesis and/or assembly of cellular metabolites (33). Several studies have reported that mitochondria serve an important role in cell reprogramming $(34,35)$. The significantly increased number and dynamic changes of the mitochondria suggest an increased requirement of energy support during cell differentiation, which may have an important role in the differentiation of human fibroblasts into neurons by small molecular compounds. The underlying mechanism requires further investigation.

It has been documented that the transcriptional program underlying embryonic neurogenesis is regulated by the spatiotemporal expression pattern of critical transcription factors $(19,36)$. Direct conversion of fibroblasts into functional neurons has been demonstrated using transcription factors $(18,37)$. NeuroD1 is a basic Helix-Loop-Helix (bHLH) transcription factor that serves an important role during neuronal differentiation, is essential for eliciting the neuronal development program and possesses the ability to reprogram other cell types into neurons $(38,39)$. Therefore, 
it may serve an important role in reprogramming fibroblasts into neurons. The expression levels of NeuroD1 transcript were only increased 3-fold in iNs compared with human fibroblasts, and this was not significantly different after 30 days of induction. It was reasoned that the neurons tend to mature after 30 days of induction, and the expression levels of transcription factors might decrease as well. This notion was supported by a previous report demonstrating that in cultured cortical neurons, expression levels of neuroD/BETA2 were decreased with increasing days in culture, as neurons became mature (40). neuroD/BETA2 is expressed highly in immature neurons (40). Indeed, the transcription of NeuroD1 was increased gradually as induction progressed, and reached a peak on day 7 , increasing as much as 4-fold compared with human fibroblasts, before it began to decline by 4 -fold on day 10 compared with human fibroblasts. In addition, NeuroD1 is able to override the presence of stimuli for pluripotency, inducing neuronal gene expression and repressing core pluripotency genes (OCT4, Klf4 and Nanog) (38). Therefore, the present study tested whether induction of iNs from HFFs using the cocktail of eight small molecular compounds bypassed the pluripotent stem stage. Results indicated that the pluripotency gene OCT4 was not induced in the iNs at any induction stage, which suggested that a direct conversion of HFFs to differentiate into iNs occurred, bypassing the pluripotent stage. In this regard, NeuroD1 may inhibit the expression of pluripotent genes and thus achieve direct trans-differentiation. Ascl1 is another bHLH factor and is a crucial regulator of neurogenesis during normal development (41). Ascl1 is a pioneering transcription factor that initiates the iN-reprogramming process by subsequently aiding other factors to secure the reprogramming route toward neuronal direction (42). In this respect, it is also a central and essential component of direct reprogramming of mouse or human fibroblasts to iNs $(37,43)$. In the present experiments, a similar trend in Ascll expression was determined. The peak expression was observed on day 7 and expression gradually decreased by 2 -fold compared with that in fibroblasts on day 30 of induction. In addition to the aforementioned findings, both bHLH factors NeuroD1 and Ascl1 were significantly elevated on day 7 of induction in the present study, which suggested that activation of bHLH factors may be a key to alter cell fate induced by the cocktail of eight small molecular compounds.

The present study has demonstrated the potential of the cocktail of eight small molecular compounds in the induction of HFF trans-differentiation into neurons. The morphology of cells was markedly altered during induction. Mechanistically, the induction of differentiation was at least in part accompanied by increased expression of endogenous neuronal transcription factors, such as the bHLH factors NeuroD1 and Ascl1, and decreased expression of fibroblast-specific genes. Further studies are required to replicate these results in other types of fibroblasts, such as skin and lung fibroblasts. Collectively, the present study suggested that the activation of specific endogenous transcription factors and cell morphology intervention by small molecular compounds further increases the induction efficiency of HFF differentiation into neurons, which may provide a novel approach for the generation of neuronal resources in cell replacement therapy of neurological disorders.

\section{Acknowledgements}

Not applicable.

\section{Funding}

The present study was supported by the Scientific Research Project of Ningxia Medical University (grant nos. XT2019024 and XY201712), the West China Top Class Discipline Project in Basic Medical Sciences, Ningxia Medical University (grant no. NXYLXK2017B07), the National Natural Science Foundation of China (grant nos. 31460300 and 81860132) and the Major Science and Technology Projects for the 13th Five-Year Plan of Ningxia Hui Autonomous Region (grant no. 2016BZ07).

\section{Availability of data and materials}

The datasets used and/or analyzed during the current study are available from the corresponding authors on reasonable request.

\section{Authors' contributions}

JY, HC, SC and QH conceived and designed the study. JY, $\mathrm{HC}, \mathrm{SG}$ and $\mathrm{HZ}$ designed the concept and performed the experiments. JY, HC, HT, LZ and ZC conducted statistical analyses, interpreted the results and searched the literature. TS collected the foreskin sample from the patient and performed cell culture. JY and QH wrote the manuscript. All authors read and approved the final manuscript.

\section{Ethics approval and consent to participate}

The present study was approved by the Ethics Review Board of Ningxia Medical University (approval no. 2015-049). Written informed consent was obtained from the participant enrolled in the present study.

\section{Patient consent for publication}

Written informed consent for publication of the study was obtained from the patient.

\section{Competing interests}

The authors declare that they have no competing interests.

\section{References}

1. Kumar A, Narayanan K, Chaudhary RK, Mishra S, Kumar S, Vinoth KJ, Padmanabhan P and Gulyás B: Current perspective of stem cell therapy in neurodegenerative and metabolic diseases. Mol Neurobiol 54: 7276-7296, 2017.

2. Chanda S, Marro S, Wernig M and Sudhof TC: Neurons generated by direct conversion of fibroblasts reproduce synaptic phenotype caused by autism-associated neuroligin-3 mutation. Proc Natl Acad Sci USA 110: 16622-16627, 2013.

3. Liu ML, Zang T and Zhang CL: Direct lineage reprogramming reveals disease-specific phenotypes of motor neurons from human ALS patients. Cell Rep 14: 115-128, 2016.

4. Sahni V and Kessler JA: Stem cell therapies for spinal cord injury. Nat Rev Neurol 6: 363-372, 2010. 
5. Takahashi K and Yamanaka S: Induction of pluripotent stem cells from mouse embryonic and adult fibroblast cultures by defined factors. Cell 126: 663-676, 2006.

6. Hyun I, Lindvall O, Ahrlund-Richter L, Cattaneo E, Cavazzana-Calvo M, Cossu G, De Luca M, Fox IJ, Gerstle C, Goldstein RA, et al: New ISSCR guidelines underscore major principles for responsible translational stem cell research. Cell Stem Cell 3: 607-609, 2008.

7. Rosemann A: Stem cell treatments for neurodegenerative diseases: Challenges from a science, business and healthcare perspective. Neurodegener Dis Manag 5: 85-87, 2015.

8. Hu W, Qiu B, Guan W, Wang Q, Wang M, Li W, Gao L, Shen L, Huang Y, Xie G, et al: Direct conversion of normal and Alzheimer's disease human fibroblasts into neuronal cells by small molecules. Cell Stem Cell 17: 204-212, 2015.

9. Li X, Zuo X, Jing J, Ma Y, Wang J, Liu D, Zhu J, Du X, Xiong L, Du Y, et al: Small-molecule-driven direct reprogramming of mouse fibroblasts into functional neurons. Cell Stem Cell 17: 195-203, 2015.

10. Pfisterer U, Ek F, Lang S, Soneji S, Olsson R and Parmar M: Small molecules increase direct neural conversion of human fibroblasts. Sci Rep 6: 38290, 2016.

11. Qin H, Zhao A, Ma K and Fu X: Chemical conversion of human and mouse fibroblasts into motor neurons. Sci China Life Sci 61: $1151-1167,2018$

12. Yang Y, Chen R, Wu X, Zhao Y, Fan Y, Xiao Z, Han J, Sun L, Wang $X$ and Dai J: Rapid and efficient conversion of human fibroblasts into functional neurons by small molecules. Stem Cell Reports 13: 862-876, 2019.

13. Livak KJ and Schmittgen TD: Analysis of relative gene expression data using real-time quantitative PCR and the 2(-Delta Delta C(T)) method. Methods 25: 402-408, 2001.

14. Huangfu D, Maehr R, Guo W, Eijkelenboom A, Snitow M, Chen AE and Melton DA: Induction of pluripotent stem cells by defined factors is greatly improved by small-molecule compounds. Nat Biotechnol 26: 795-797, 2008

15. Ladewig J, Mertens J, Kesavan J, Doerr J, Poppe D, Glaue F, Herms S, Wernet P, Kögler G, Müller FJ, et al: Small molecules enable highly efficient neuronal conversion of human fibroblasts. Nat Methods 9: 575-578, 2012.

16. Madhu V, Dighe AS, Cui Q and Deal DN: Dual inhibition of activin/Nodal/TGF- $\beta$ and BMP signaling pathways by SB431542 and dorsomorphin induces neuronal differentiation of human adipose derived stem cells. Stem Cells Int 2016: 1035374, 2016.

17. Lamas NJ, Johnson-Kerner B, Roybon L, Kim YA, Garcia-Diaz A, Wichterle $\mathrm{H}$ and Henderson CE: Neurotrophic requirements of human motor neurons defined using amplified and purified stem cell-derived cultures. PLoS One 9: e110324, 2014.

18. Li W, Zhou H, Abujarour R, Zhu S, Young Joo J, Lin T, Hao E, Schöler HR, Hayek A and Ding S: Generation of human-induced pluripotent stem cells in the absence of exogenous Sox 2. Stem Cells 27: 2992-3000, 2009.

19. Ambasudhan R, Talantova M, Coleman R, Yuan X, Zhu S, Lipton SA and Ding S: Direct reprogramming of adult human fibroblasts to functional neurons under defined conditions. Cell Stem Cell 9: 113-118, 2011.

20. Guillemot F: Spatial and temporal specification of neural fates by transcription factor codes. Development 134: 3771-3780, 2007.

21. Pang ZP, Yang N, Vierbuchen T, Ostermeier A, Fuentes DR, Yang TQ, Citri A, Sebastiano V, Marro S, Südhof TC and Wernig M: Induction of human neuronal cells by defined transcription factors. Nature 476: 220-223, 2011.

22. Hou PS, Chuang CY, Yeh CH, Chiang W, Liu HJ, Lin TN and Kuo HC: Direct conversion of human fibroblasts into neural progenitors using transcription factors enriched in human ESC-Derived neural progenitors. Stem Cell Reports 8: 54-68, 2017.

23. Kim SM, Flasskamp H, Hermann A, Araúzo-Bravo MJ, Lee SC, Lee SH, Seo EH, Lee SH, Storch A, Lee HT, et al: Direct conversion of mouse fibroblasts into induced neural stem cells. Nat Protoc 9: 871-881, 2014

24. Caiazzo M, Giannelli S, Valente P, Lignani G, Carissimo A, Sessa A, Colasante G, Bartolomeo R, Massimino L, Ferroni S et al: Direct conversion of fibroblasts into functional astrocytes by defined transcription factors. Stem Cell Reports 4: 25-36, 2015
25. Biswas D and Jiang P: Chemically induced reprogramming of somatic cells to pluripotent stem cells and neural cells. Int J Mol Sci 17: 226, 2016.

26. Fritz AL, Adil MM, Mao SR and Schaffer DV: cAMP and EPAC signaling functionally replace OCT4 during induced pluripotent stem cell reprogramming. Mol Ther 23: 952-963, 2015.

27. Hou P, Li Y, Zhang X, Liu C, Guan J, Li H, Zhao T, Ye J, Yang W, Liu K, et al: Pluripotent stem cells induced from mouse somatic cells by small-molecule compounds. Science 341: 651-654, 2013.

28. Ichida JK, Blanchard J, Lam K, Son EY, Chung JE, Egli D, Loh KM, Carter AC, Di Giorgio FP, Koszka K, et al: A small-molecule inhibitor of tgf-Beta signaling replaces sox 2 in reprogramming by inducing nanog. Cell Stem Cell 5: 491-503, 2009.

29. Sato N, Meijer L, Skaltsounis L, Greengard $P$ and Brivanlou AH: Maintenance of pluripotency in human and mouse embryonic stem cells through activation of Wnt signaling by a pharmacological GSK-3-specific inhibitor. Nat Med 10: 55-63, 2004.

30. Chapman S, Liu X, Meyers C, Schlegel R and McBride AA: Human keratinocytes are efficiently immortalized by a Rho kinase inhibitor. J Clin Invest 120: 2619-2626, 2010.

31. Huangfu D, Osafune K, Maehr R, Guo W, Eijkelenboom A, Chen S, Muhlestein W and Melton DA: Induction of pluripotent stem cells from primary human fibroblasts with only Oct 4 and Sox2. Nat Biotechnol 26: 1269-1275, 2008.

32. Yoshida Y, Takahashi K, Okita K, Ichisaka T and Yamanaka S: Hypoxia enhances the generation of induced pluripotent stem cells. Cell Stem Cell 5: 237-241,2009.

33. Dimmer KS and Scorrano L: (De)constructing mitochondria: What for? Physiology (Bethesda) 21: 233-241, 2006

34. Prieto J, Leon M, Ponsoda X, García-García F, Bort R, Serna E, Barneo-Muñoz M, Palau F, Dopazo J, López-García C and Torres J: Dysfunctional mitochondrial fission impairs cell reprogramming. Cell Cycle 15: 3240-3250, 2016.

35. Prieto J, Leon M, Ponsoda X, Sendra R, Bort R, Ferrer-Lorente R, Raya A, López-García C and Torres J: Early ERK1/2 activation promotes DRP1-dependent mitochondrial fission necessary for cell reprogramming. Nat Commun 7: 11124, 2016.

36. Spitz F and Furlong EE: Transcription factors: From enhancer binding to developmental control. Nat Rev Genet 13: 613-626, 2012.

37. Vierbuchen T, Ostermeier A, Pang ZP, Kokubu Y, Sudhof TC and Wernig M: Direct conversion of fibroblasts to functional neurons by defined factors. Nature 463: 1035-1041, 2010.

38. Pataskar A, Jung J, Smialowski P, Noack F, Calegari F, Straub T and Tiwari VK: NeuroD1 reprograms chromatin and transcription factor landscapes to induce the neuronal program. EMBO J 35: 24-45, 2016.

39. Aprea J, Nonaka-Kinoshita M and Calegari F: Generation and characterization of Neurod1-CreER(T2) mouse lines for the study of embryonic and adult neurogenesis. Genesis 52: 870-878, 2014.

40. Lee JK, Cho JH, Hwang WS, Lee YD, Reu DS and Suh-Kim H: Expression of neuroD/BETA2 in mitotic and postmitotic neuronal cells during the development of nervous system. Dev Dyn 217: 361-367, 2000.

41. Imayoshi I and Kageyama R: bHLH factors in self-renewal, multipotency, and fate choice of neural progenitor cells. Neuron 82: 9-23, 2014.

42. Wapinski OL, Vierbuchen T, Qu K, Lee QY, Chanda S, Fuentes DR, Giresi PG, Ng YH, Marro S, Neff NF, et al: Hierarchical mechanisms for direct reprogramming of fibroblasts to neurons. Cell 155: 621-635, 2013.

43. Shi Z, Zhang J, Chen S, Li Y, Lei X, Qiao H, Zhu Q, Hu B, Zhou Q and Jiao J: Conversion of fibroblasts to parvalbumin neurons by one transcription factor, Ascl1, and the chemical compound forskolin. J Biol Chem 291: 13560-13570, 2016.

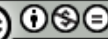

This work is licensed under a Creative Common Attribution-NonCommercial-NoDerivatives 4.0 International (CC BY-NC-ND 4.0) License. 